\title{
Implications Of Burst Oscillations From IGR J17480-2446
}

\section{Yuri Cavecchi*}

A. Pannekoek Institute, University of Amsterdam

E-mail: y.cavecchi@uva.nl

\section{A. Patruno ${ }^{1}$, B. Haskell ${ }^{1}$, A.L. Watts ${ }^{1}$, Y. Levin ${ }^{3}$, M. Linares ${ }^{4}$, D. Altamirano ${ }^{1}$, R.} Wijnands ${ }^{1}$, M. v.d. Klis ${ }^{1}$

${ }^{1}$ A. Pannekoek, University of Amsterdam, ${ }^{2}$ Sterrewacht Leiden, University of Leiden, ${ }^{3}$ School of Physics, Monash Univerity, ${ }^{\mathbf{4}}$ MIT Kavli Institute for Astrophysics and Space

The recently-discovered accreting X-ray pulsar IGR J17480-2446 spins at a frequency of $\sim 11$ $\mathrm{Hz}$ and shows Type I burst oscillations at the same frequency. Such oscillations cannot be caused by global modes in the neutron star ocean or by a Coriolis force confined hot spot. The most likely scenario is that the burst oscillations are produced by a hot spot confined by hydromagnetic stresses.

Fast X-ray timing and spectroscopy at extreme count rates: Science with the HTRS on the International $X$-ray Observatory - HTRS2011,

February 7-11, 2011

Champéry, Switzerland

\footnotetext{
* Speaker.
} 


\section{Introduction}

Accreting neutron stars (NSs) in low mass X-ray binaries show bright Type I X-ray bursts which begin with a rapid increase of the X-ray flux (the rise) followed by a slow decrease (the tail) to the pre-burst luminosity and last $\sim 10-100 \mathrm{~s}$. These bursts are powered by thermonuclear runaways and a significant fraction of them display quasi-periodic modulations, known as burst oscillations (BOs).

$\mathrm{BO}$ frequencies are linked to the spin frequencies of the neutron stars. This has been confirmed in five accretion-powered millisecond X-ray pulsars (AMXPs), where it was found that BO frequencies are within a few percent of the spin frequencies (Strohmayer \& Bildsten, 2003). This implies that BOs are caused by a near-stationary temperature asymmetry which persists in the surface layers of the star during the burst.

The detailed phenomenology of BOs, however, is diverse. In the sources which have evidence for substantial magnetic fields (the persistent AMXPs), for example, BOs appear at a near constant frequency in the tail, with some fast chirps in the rise. In the intermittent AMXPs, and NSs without evidence for a dynamically important magnetic field, BOs typically drift upwards by a few Hertz during the burst. Both the origin of the surface temperature asymmetry that causes the BOs, and the reason for the observed frequency drifts, remain unsolved puzzles.

One possibility is that the asymmetry is caused by global modes (waves) that develop in the bursting ocean (Heyl, 2004). As the ocean cools, its scale height $H$ decreases and the pattern speed, which scales as $\sqrt{H}$, changes, leading to frequency drift. Different kind of modes are possible in the NS ocean, but none of the models proposed have managed to explain both the observed frequencies and the magnitude of the drifts.

An alternative possibility is that a compact burning hot spot develops on the surface. The question is then how confinement might be achieved. For unmagnetized stars, the Coriolis force could be an effective confining mechanism (Spitkovsky et al., 2002). Although lifting and expansion of the hot fluid should cause spreading of the burning fuel, the Coriolis force would oppose such motion of the flame front by deflecting its velocity. This mechanism could account for the presence of oscillations in the burst rise, although it does not easily explain the presence of BOs in the tail or the frequency drifts. The Coriolis force was attractive in that it could explain why BOs had not been seen in any NS with spin frequency $v_{\mathrm{s}} \lesssim 245 \mathrm{~Hz}$ (since for more slowly rotating stars the Coriolis force is not dynamically relevant).

A strong magnetic field could also lead to a confined hot spot, with the restoring force supplied by field pressure or stress (see Brown \& Bildsten, 1998). This mechanism is particularly plausible for accreting pulsars, where the existence of dynamically important magnetic fields is suggested by the presence of accretion-powered pulsations (APPs).

\section{Observations}

IGR J17480 is an $11 \mathrm{~Hz}$ pulsar in a $21.3 \mathrm{hr}$ orbit around a companion with $M>0.4 M_{\odot}$ (Strohmayer \& Markwardt, 2010; Papitto et al., 2011). We used all RXTE/PCA public observations of J17480 taken between MJD 55482.0 and 55519.2 (Fig. 1). Bursts were identified visually in a $1 \mathrm{~s}$ lightcurve. The beginning and end of the bursts were defined as the points where the flux first 


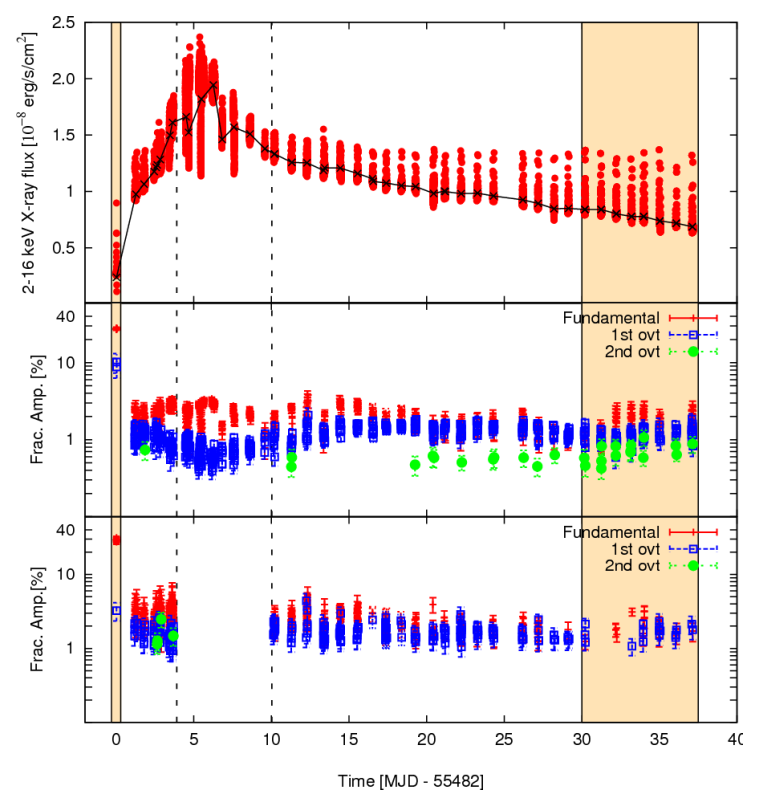

Figure 1: Top panel: Outburst lightcurve. Middle Panel: Accretion powered pulsation (APP) fractional amplitude. Lower Panel: Burst oscillation (BO) fractional amplitude. The phases of the two sets of oscillations are coincident within $\sim 0.05$ cycles.

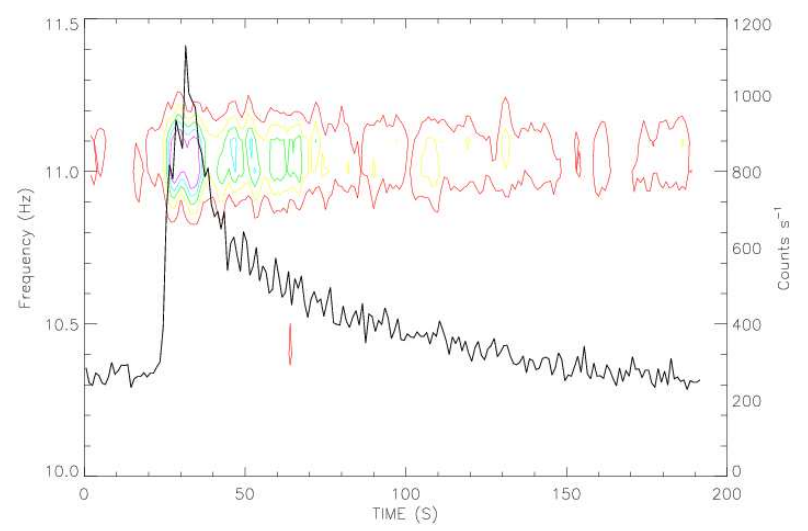

Figure 2: First burst dynamical power spectrum showing strong BOs. Leahy normalized power contours are for 30, 60, 90, 120 and 150. Peak fractional amplitude is $\sim 30 \%$. The frequencies of APPs and BOs are the same within $10^{-4} \mathrm{~Hz}$.

and last exceeded the maximum pre-burst flux in the same observation. Typical burst duration is $\sim 100-200 \mathrm{~s}$.

Burst oscillations were detected in all the 231 bursts we identified, but only pulse harmonics with SNR $>3.5$ were retained. The fractional amplitudes of BOs are always of the same order or larger than those of APPs, and always significantly larger than expected if the modulations stem only from residual persistent emission.

The pulse frequency and derivative obtained for BOs and APPs are consistent with being the same to within two standard deviations. Moreover, adjacent BOs and APPs are always phase coincident and phase locked to within $\sim 0.05$ cycles. For example, in the first observations, when the $\mathrm{BO}$ and 
APP amplitudes are around 30\% (Fig. 1) and we have the highest SNR, the frequencies of APPs $(11.044881(2) \mathrm{Hz})$ and BOs $(11.04493(7) \mathrm{Hz})$ are identical within the errors $\left(\sim 7 \times 10^{-5} \mathrm{~Hz}\right)$ and the phase difference between the peaks of the fundamental for APPs and BOs is $0.014 \pm 0.005$ cycles $(5.1 \pm 1.8 \mathrm{deg})$.

\section{Interpretation}

\subsection{What does not work: global modes and Coriolis confined hot spot}

First of all, we can prove that models involving global modes cannot explain the presence of the observed BOs: in general, the observed frequency $\left(v_{o}\right)$ is related to the spin $\left(v_{s}\right)$ and mode $\left(v_{r}\right)$ ones as

$$
v_{o}=m v_{s}+v_{r}
$$

Where $m$ is the azimuthal number. Modes with $m>2$ have fractional amplitudes that are too small to give the high powers we see in the power spectra (Heyl, 2004). As for modes with $m=0$, that would imply $v_{r}-v_{o} \sim 10^{-4} \mathrm{~Hz}$, but no mechanism can tie the mode frequency to the spin one so closely, without requiring an extreme fine tuning. Finally, in the case $m=1$, we would have $v_{r} \sim 10^{-4} \mathrm{~Hz}$, but this implies that the time scale exciting the mode $\left(\sim 1 / v_{r}\right)$ is $\sim 10^{4} \mathrm{~s}$, which is too long compared to the bursts themselves.

Likewise, we can exclude a Coriolis confined hot spot as an explanation, because for the Coriolis force to be dynamically effective the spin frequency should be $v_{s} \gtrsim 100 \mathrm{~Hz}$, which is an order of magnitude higher than the case of J17480. Indeed, the fluid is confined within a distance of order the Rossby radius (Spitkovsky et al., 2002)

$$
R=\sqrt{g H} / 4 \pi v_{\mathrm{s}}
$$

Assuming a mass of $1.4 \mathrm{M}_{\odot}$, a radius of $10 \mathrm{~km}$ and a scale height for the burning fluid $H$ of $10 \mathrm{~m}$ (Spitkovsky et al., 2002), $R$ is $\sim 34 \mathrm{~km}$ in the case of J17480 and this is $\sim 3$ times as much as the actual radius of the star, implying no confinement at all.

\subsection{What does work: magnetically confined hot spot}

Finally, we can prove that a confining magnetic field is compatible with the observational constraints: a first limit on the strength of the magnetic field comes from the requirement that the magnetospheric radius (the radius within which the dynamic of the accretion disc starts being dominated by the magnetic field of the NS) be outside the star (otherwise the accretion would not be channeled on the magnetic poles, but rather happen via a boundary layer around the equator of the star, Psaltis \& Chakrabarty, 1999). A second requirement is that the the corotation radius (the radius from which the disc begins spinning slower than the star) must be greater than the magnetospheric radius (otherwise the system would be in the propeller status, i.e. matter is expelled instead of being accreted, Psaltis \& Chakrabarty, 1999). These two combined conditions require that

$$
2 \times 10^{7} \mathrm{G} \lesssim B \lesssim 10^{10} \mathrm{G}
$$


for J17480.

As for the magnetic field required to confine a hot spot, the magnetic tension needs being able to compensated the pressure of the fluid. The seed field sufficient to statically confine the accreted fluid at the magnetic pole as deep as the ignition depth is of order (Brown \& Bildsten, 1998)

$$
B \gtrsim 3 \times 10^{10} \mathrm{G}
$$

whereas, if the fluid spreads before the compression of magnetic lines leads to a dynamically important tension, the sufficient seed field is an order of magnitude less (Heng \& Spitkovsky, 2009)

$$
B \gtrsim 4 \times 10^{9} \quad \mathrm{G}
$$

which is compatible with the constraints derived from the accretion process. We conclude that the magnetic field can confine the burning fluid, thereby explaining the presence of BOs in J17480.

\section{Conclusions}

The observational constraints on the magnetic field of J17480 and the requirements for confinement of a hot spot are compatible: the magnetic field is capable of confining a hot spot, while the other proposed explanation for BOs (global modes and Coriolis force confinement) are not applicable to this source.

Moreover, a burning hot spot at the same position as the magnetically channeled accretion location can naturally explain the observed features and similarities between BOs and APPs (see Fig. 2), in particular the phase coincidence and the fact that the fractional amplitude of the BOs tracks that of the APPs.

A question remains about the mechanism for BOs in sources with an apparently weaker magnetic field, the non pulsating sources and the intermittent pulsars: a different mechanism may be required in those cases. Global modes or Coriolis confinement could still be the explanation and the wider emitting surface involved, or the involvement of higher colatitudes, could explain some observational differences such as the suppression of higher harmonics.

\section{References}

Strohmayer, T., \& Bildsten, L. 2003, ArXiv Astrophysics 0301544

Heyl, J. S. 2004, ApJ, 600, 939

Spitkovsky, A., Levin, Y., \& Ushomirsky, G. 2002, ApJ, 566, 1018

Brown, E. F., \& Bildsten, L. 1998, ApJ, 496, 915

Strohmayer, T. E., \& Markwardt, C. B. 2010, The Astronomer's Telegram, 2929, 1

Papitto, A., D’Aì, A., Motta, S., Riggio, A., Burderi, L., di Salvo, T., Belloni, T., \& Iaria, R. 2011, A\&A, 526, L3+

Psaltis, D., \& Chakrabarty, D. 1999, ApJ, 521, 332

Heng, K., \& Spitkovsky, A. 2009, ApJ, 703, 1819 